\title{
Role of Factor Xa Inhibitors in Cancer-Associated Thrombosis: Any New Data?
}

\author{
Ali Zalpour, ${ }^{1}$ Michael H. Kroll, ${ }^{2}$ Vahid Afshar-Kharghan, ${ }^{2}$ Syed Wamique Yusuf, ${ }^{3}$ \\ and Carmen Escalante ${ }^{4}$ \\ ${ }^{1}$ Pharmacy Clinical Programs, Division of Pharmacy, The University of Texas MD Anderson Cancer Center, Houston,
TX 77030, USA
${ }^{2}$ Section of Benign Hematology, The University of Texas MD Anderson Cancer Center, Houston, TX 77030, USA
${ }^{3}$ Department of Cardiology, The University of Texas MD Anderson Cancer Center, Houston, TX 77030, USA
${ }^{4}$ Department of General Internal Medicine, The University of Texas MD Anderson Cancer Center, Houston, TX 77030, USA
}

Correspondence should be addressed to Ali Zalpour, azalpour@mdanderson.org

Received 21 July 2011; Revised 10 August 2011; Accepted 11 August 2011

Academic Editor: Shaji Kumar

Copyright (C) 2011 Ali Zalpour et al. This is an open access article distributed under the Creative Commons Attribution License, which permits unrestricted use, distribution, and reproduction in any medium, provided the original work is properly cited.

\begin{abstract}
The association between cancer and venous thromboembolism (VTE) has been well documented in the literature. Prevention and treatment of VTE in cancer patients is imperative. Typically, the mainstay regimen for VTE prevention and treatment has been anticoagulation therapy, unless contraindicated. This therapy consists of unfractionated heparin (UFH), low-molecular-weight heparin (LMWH), factor Xa inhibitor, or vitamin K antagonist (VKA). Current guidelines recommend LMWH over VKA for the treatment of VTE in cancer patients. Factor-specific anticoagulants have been proven safe and effective, and recently factor Xa inhibitors have emerged as a treatment alternative to heparins and VKA. Currently, three factor Xa inhibitors have been identified: fondaparinux (the only one approved so far by the US Food and Drug Administration), idraparinux (in clinical trials), and idrabiotaparinux (in clinical trials). This paper will examine the role of these agents, focusing on fondaparinux, for the prevention and treatment of VTE in cancer patients.
\end{abstract}

\section{Introduction}

The association between cancer and venous thromboembolism (VTE) has been well recognized and established [1]. Cancer patients have a 4 -fold higher risk of developing VTE than do patients without cancer, and chemotherapy increases that risk to 6-fold [2]. In cancer patients undergoing surgical procedures, rates of postoperative VTE can increase 2-fold greater than rates of postoperative VTE in patients without cancer [3]. Frequency of VTE has increased by up to $28 \%$ in years 1995 to 2003 in hospitalized cancer patients and with the higher mortality rates compared to those hospitalized cancer patients without VTE $(16.3 \%$ versus $6.3 \%, P<$ 0.0001) [4].

Given that the 1-year survival rate in cancer patients with VTE is much lower than in cancer patients without VTE (12\% versus $36 \%$ ), appropriate and effective thromboprophylaxis_-both pharmacologic and nonpharmacologic-is imperative [9]. Effective thromboprophylaxis can minimize mortality and morbidity, potentially affect survival, and lower health-care costs associated with VTE.

The National Comprehensive Cancer Network (NCCN), the American Society of Clinical Oncology (ASCO), and recently the American College of Chest Physicians (ACCP) have published guidelines for the prevention and treatment of VTE in cancer patients (Table 1). These guidelines recommend using unfractionated heparin (UFH), low-molecularweight heparins (LMWHs), and, recently, direct factor Xa inhibitors for the prevention of VTE in cancer patients who are hospitalized [5-8].

Currently, prophylaxis for catheter-related thrombosis is not recommended owing to conflicting evidence and a lack of major clinical trials [10]. For treatment of VTE in cancer patients, guidelines recommend LMWH as first-line therapy and vitamin $\mathrm{K}$ antagonist (VKA) as second-line therapy. The recommended duration of treatment ranges 
TABLE 1: Summary of guidelines for prevention and treatment of venous thromboembolism in cancer [5-8].

\begin{tabular}{|c|c|c|c|c|}
\hline Guidelines and pharmacologic prophylaxis & VKA & UFH & LMWH & FXa-I \\
\hline $\begin{array}{l}\text { American College of Chest Physicians (ACCP) } \\
\text { (Prevention in cancer patients (medical and surgical)) }\end{array}$ & No & Yes (SQ) & Yes & Yes \\
\hline
\end{tabular}

Duration of prevention: (1) For medical oncology cancer patients who have acute medical illness or who are bedridden for the duration of hospitalization. (2) For surgical cancer patients (pelvic, abdominal, orthopedic) duration of prophylaxis up to 4 weeks. (3) In the presence of contraindications or high risk of bleeding, mechanical methods may be temporarily substituted and pharmacologic prophylaxis should resume after risk of bleeding subsides.

\begin{tabular}{|c|c|c|c|c|c|}
\hline American College of Chest Physician (ACCP) & Acute & No & No & Yes & Not addressed \\
\hline (Treatment in cancer patients) & Long term & Yes & No & Yes & No \\
\hline
\end{tabular}

Duration of treatment: At least 3 months of treatment with LMWH, followed by treatment with either LMWH or VKA.

American Society of Clinical Oncology (ASCO)

(Prevention in cancer patients)

No $\quad$ Yes $(\mathrm{SQ}) \quad$ Yes $\quad$ Yes

Duration of prevention: (1) For as long as the patient is hospitalized (due to surgery or acute medical illness) or until the patient is ambulatory. (2) In the presence of contraindication or high risk of bleeding, mechanical methods may be temporarily substituted and pharmacologic prophylaxis should resume after risk of bleeding subsides. (3) In certain multiple myeloma patients receiving thrombogenic chemotherapy (lenalidomide or thalidomide with dexamethasone), low-dose VKA (INR 1.5) or enoxaparin (40 mg) may be considered.

\begin{tabular}{lccccc}
\hline American Society of Clinical Oncology (ASCO) & Acute 5-10 days & No & Yes (IV) & Yes & Yes \\
(Treatment in cancer patients) & Long term & Yes & No & Yes & Not addressed \\
\hline
\end{tabular}

Duration of treatment: LMWH is preferred for 5-10 days, then LMWH for at least 6 months. VKA may be substituted if LMWH is not accessible. After 6 months of treatment, indefinite treatment duration for cancer patients with metastasis or those actively receiving chemotherapy.

National Comprehensive Cancer Network (NCCN)

(Prevention in cancer patients)

Yes $\quad$ Yes $(S Q) \quad$ Yes $\quad$ Yes

Duration of prevention: (1) For the duration of hospitalization for medical illness and up to 4 weeks in surgical cancer patients. (2) In the presence of contraindication, use mechanical prophylaxis until bleeding risk subsides. (3) In certain high-risk medical oncology patients (i.e., aggressive tumor such as pancreatic, gastric, lymphoma, or in cases of obesity or prior VTE), longer prophylaxis is recommended. (4) In certain multiple myeloma patients receiving thrombogenic chemotherapy (lenalidomide or thalidomide with dexamethasone), VKA (INR of 2-3) or aspirin (81-325 mg) may be considered.

\begin{tabular}{lccccc}
\hline National Comprehensive Cancer Network (NCCN) & Acute 5-10 days & No & Yes (IV) & Yes & Yes \\
(Treatment in cancer patients) & Long term & Yes & No & Yes & Not addressed \\
\hline
\end{tabular}

Duration of treatment: (1) LMWH is preferred for the first 3-6 months in DVT and 6-12 months in PE. (2) VKA can be considered if LMWH is not accessible.

VKA: vitamin K antagonist; UHF: unfractionated heparin; LMWH: low-molecular-weight heparin; FXa-I: direct factor-Xa inhibitor; SQ: subcutaneous; IV: intravenous; INR: international normalized ratio; DTV: deep vein thrombosis.

from 3 to 12 months, depending on which set of guidelines is followed. For long-term treatment of VTE in cancer patients, guidelines do not address the role of factor Xa inhibitors [58].

Factor-specific anticoagulants have been proven safe and effective, and, recently, factor Xa inhibitors have emerged as an alternative therapy for VTE in cancer patients. In this paper, we examine the role of injectable factor Xa inhibitors in cancer patients, focusing on fondaparinux, the only such agent yet approved by the US Food and Drug Administration (FDA). The paper covers clinical pharmacology, clinical pharmacokinetics, and available data from clinical trials in cancer patients. We also addressed the safety of using factor Xa inhibitors for the prevention and long-term treatment of VTE in special populations of cancer patients, such as patients who are obese, have renal insufficiency, or have a high risk of bleeding. Finally, we presented recent and emerging data on idraparinux and idrabiotaparinux, two factor Xa inhibitors currently being investigated in clinical trials for the prevention and treatment of VTE in cancer patients.

\section{Fondaparinux}

2.1. Pharmacodynamic Profile of Indirect Factor Xa Inhibitor. After activation of the coagulation cascade by either the intrinsic or extrinsic pathways, the generation of factor $\mathrm{Xa}$ is amplified. Factor Xa is the first step in the common pathway that leads to the generation of thrombin which converts prothrombin to thrombin and finally the formation of the fibrin (Figure 1) [11]. Briefly, VKAs inhibit the formation of factors II, VII, IX, X and the natural anticoagulant proteins $\mathrm{C}$ and S [12]. UFH and LMWHs have more specificity for inhibition of coagulation factors such as factor Xa and factor IIa. LMWH has been shown to bind mostly to factor Xa and 


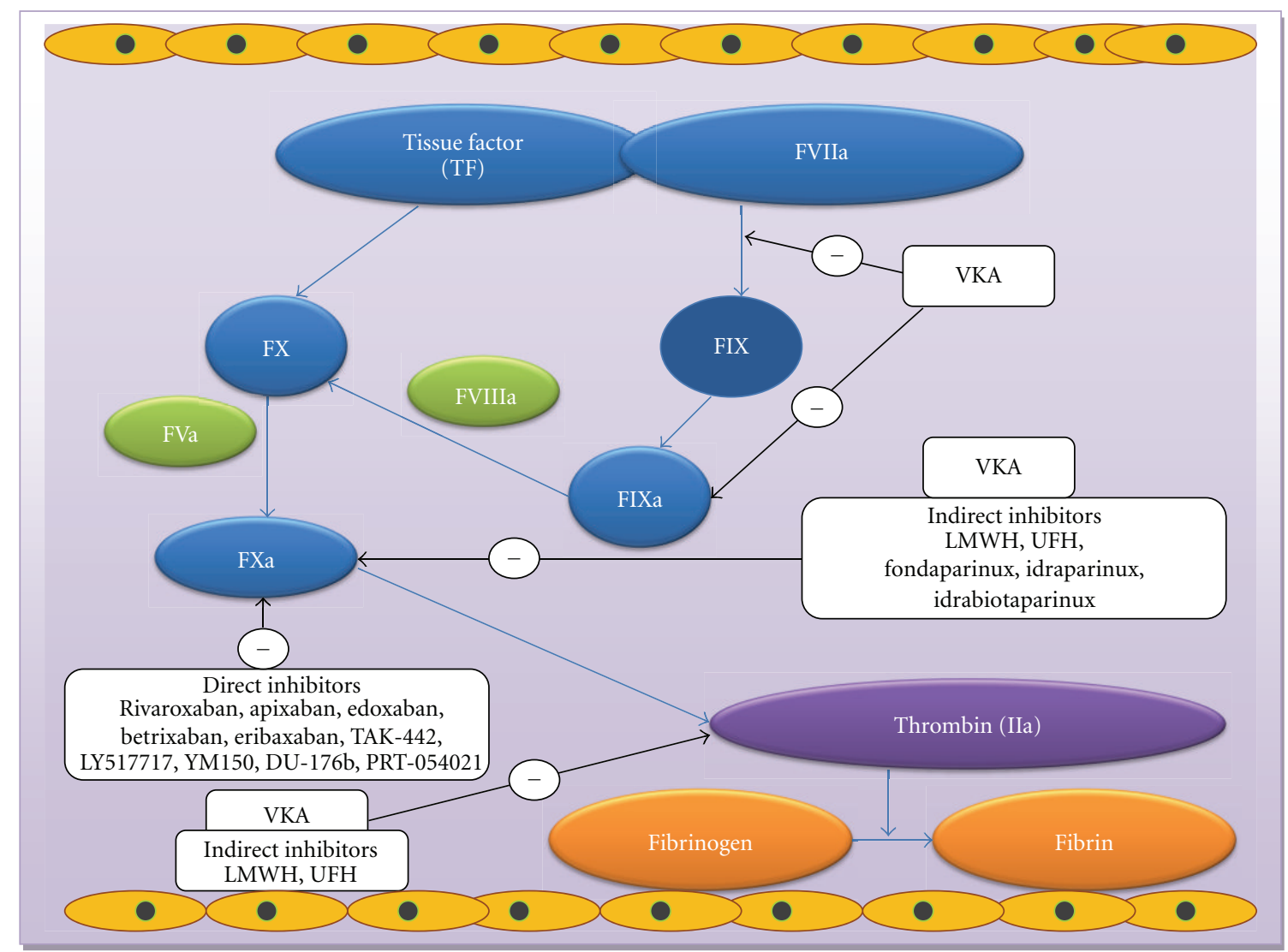

FIGURE 1: Mechanism of action of anticoagulants. Abbreviations: vitamin k antagonist (VKA); low-molecular-weight heparin (LMWH); unfractioned heparin (UFH).

minimally to factor IIa through activation of antithrombin (AT) III (ATIII) [12].

In recent years, more targeted anticoagulants such as fondaparinux (1728 Da or pentasaccharides) have emerged that specifically target factor Xa. Fondaparinux does not inhibit factor IIa since more than 18 monosaccharides are required for simultaneous binding to AT and factor IIa [13]. Fondaparinux binds reversibly to AT in a $1: 1$ ratio through a noncovalent bond, which causes conformational change and then selectively and rapidly inhibits factor Xa and thereby inhibition of thrombin (inhibition of 1 unit of factor Xa results in $\sim 50$ units lower concentration of thrombin). Fondaparinux binding to AT causes an approximate 300 -fold increase in the rate at which AT inhibits factor Xa $[14,15]$. Direct factor Xa inhibitors inhibit free factor Xa and bound factor $\mathrm{Xa}$ in the prothrombinase complex, whereas indirect factor Xa (fondaparinux) inhibitors target factor Xa through AT. Factor Xa inhibitors can block the formation of thrombin rather than the inhibition of thrombin activity [16].

Currently, there are three injectable indirect factor Xa inhibitors in various stages of development: fondaparinux, idraparinux, and idrabiotaparinux (Table 2). Only fondaparinux has been approved by the FDA for the prevention and treatment of VTE $[17,18]$.

Presently, there are oral formulations of direct factor Xa inhibitors, but these are beyond the scope of this paper; oral factor Xa inhibitors that are in various phases of clinical trials include rivaroxaban (Xarelto), which has been approved in Europe for prevention of VTE, apixaban, edoxaban, betrixaban, eribaxaban, TAK-442, LY517717, YM150, DU176b, and PRT-054021 [19].

2.2. Pharmacokinetic Profile. Fondaparinux exhibits the following pharmacokinetic parameters: complete bioavailability of $\sim 100 \%$; peak plasma concentration of $0.34 \mathrm{mg} / \mathrm{L}$ (dose of $2.5 \mathrm{mg}$ ); time-to-peak plasma concentration of 1.7 hours; linear pharmacokinetics in the $2 \mathrm{mg}$ to $8 \mathrm{mg}$ dose range. Fondaparinux is excreted via the kidneys (64\% to $74 \%$ ) with a terminal half-life $\left(T_{\beta 1 / 2}\right)$ of $\sim 17$ hours [20]. Fondaparinux is not metabolized by the cytochrome P450 (CYP) enzyme in the liver [15].The volume of distribution ( $\mathrm{Vd}$ ) and protein binding of fondaparinux is estimated to be from $7 \mathrm{~L}$ to $11 \mathrm{~L}$ and $>97 \%$, respectively; hence, fondaparinux does not distribute to extravascular space such as adipose tissue. Fondaparinux has a negligible nonspecific binding to $\alpha{ }_{-1}$ acid-glycoprotein [15].

Pharmacokinetic studies have produced peak and trough levels of fondaparinux at steady state $\left(C_{\mathrm{ss} \max / \min }\right)$ of 0.39 to $0.50 \mathrm{mcg} / \mathrm{mL}$ and 0.14 to $0.19 \mathrm{mcg} / \mathrm{mL}$ for the $2.5-\mathrm{mg}$ dosing schedule, respectively. The peak and trough levels (or ranges) of fondaparinux at $C_{\mathrm{ss}} \max / \mathrm{min}$ for the 5-mg, 7.5-mg, and 10$\mathrm{mg}$ dosing schedules are 1.20 to $1.26 \mathrm{mcg} / \mathrm{mL}$ and 0.46 to $0.62 \mathrm{mcg} / \mathrm{mL}[18]$. 
TABLE 2: Injectable factor Xa inhibitors $[17,18]$.

\begin{tabular}{|c|c|c|c|}
\hline & Fondaparinux & Idraparinux & Idrabiotaparinux \\
\hline Target & Factor Xa & Factor Xa & Factor Xa \\
\hline Route of administration & Subcutaneous & Subcutaneous & Subcutaneous \\
\hline Prevention dose & $2.5 \mathrm{mg}$ & Not available & Not available \\
\hline Dosing schedule & Daily & Weekly & Weekly \\
\hline Therapeutic dose & $\begin{array}{c}\text { Acute VTE in conjunction with VKA: } \\
\text { (ASCO) + (NCCN) } \\
50 \mathrm{~kg}: 5 \mathrm{mg} \text { once daily; } 50-100 \mathrm{~kg}: 7.5 \mathrm{mg} \text { once daily; } \\
>100 \mathrm{~kg}: 10 \mathrm{mg} \text { once daily }\end{array}$ & $2.5 \mathrm{mg}$ & $3.0 \mathrm{mg}$ \\
\hline Bioavailability (\%) & 100 & 100 & 100 \\
\hline Half-life $\left(t_{1 / 2}\right)$ & 17 hours & 60 days & 60 days \\
\hline Elimination & Renal & Renal & Renal \\
\hline Antidote & None & None & Avidin \\
\hline Market status/FDA approval & $\begin{array}{l}\text { FDA approved for prevention and treatment of venous } \\
\text { thromboembolism }\end{array}$ & In clinical trials & In clinical trials \\
\hline Contraindication & $\begin{array}{ll}\text { (i) Patients with active bleeding } & \text { (i) Patients with } \mathrm{CrCl} \\
\text { (ii) Bacterial endocarditis } & <30 \mathrm{~mL} / \mathrm{min} \\
\text { (iii) Thrombocytopenia } & \text { (ii) Neuraxial anesthesia } \\
\text { associated with positive } & \text { (iii) Weight }<50 \mathrm{~kg} \\
\text { antiplatelet antibodies } & \text { (prophylactic doses) }\end{array}$ & Not available & Not available \\
\hline Monitoring parameters & Monitor hemoglobin, platelets & Not available & Not available \\
\hline
\end{tabular}

VTE: venous thromboembolism; VKA: vitamin K antagonist; ASCO: American Society of Clinical Oncology; NCCN: National Comprehensive Cancer Network; FDA: US Food and Drug Administration; CrCl: creatinine clearance.

2.3. Comparative Efficacy in VTE Prevention Trials. Primary data using fondaparinux for prevention of VTE in cancer patient is clearly lacking. In a study of elderly patients (number $(n)=849)$ with acute medical illnesses $(\sim 15 \%$ had cancer), patients who received fondaparinux (2.5 mg per day per day for 14 days) had a relative risk reduction (RRR) of $46 \%$ (95\% confidence interval (CI): $7.7 \%$ to $69.3 \%$ ) in VTE compared with patients who received the placebo $(5.6 \%$ versus $10.5 \%)$. The patients who received fondaparinux had a $0.4 \%$ incidence of major bleeding, and their 32-day mortality rate was $3.3 \%$, while patients who received the placebo had a 32 -day mortality rate of $6.0 \%(P=0.006)$. In this study, fondaparinux provided the same efficacy across body weight ranges of $32 \mathrm{~kg}$ to $111 \mathrm{~kg}$, and bleeding was not related to body weight [21].

Turpie et al. showed a VTE rate reduction of $69.8 \%$ in patients who underwent major abdominal surgery $(40 \%$ of patients had surgery for cancer); patients received either fondaparinux (2.5 mg per day or prophylactic dose) plus intermittent pneumatic compression (IPC) or IPC alone, with low major bleeding rates of $1.6 \%$ bleeding rate in the fondaparinux plus IPC group and the $0.2 \%$ in the
IPC alone group $(P=0.006)$ [22]. The first injection of fondaparinux was given 6 to 8 hours after surgical closure, and the second injection of fondaparinux was given 16 to 28 hours after the first injection; an epidural, if used, was removed 2 hours prior to the first injection. In this study, the efficacy of fondaparinux was proven irrespective of age, gender, weight (mean, $82 \mathrm{~kg}$ ), or type and duration of surgery. The overall mortality rate was $1.3 \%$ in the fondaparinux plus IPC group (1 fatal pulmonary embolism $(\mathrm{PE})$ ) and $0.8 \%$ in the IPC group ( 1 fatal PE, $P=0.42$ ) [22].

In another study of VTE prevention in surgery patients, Agnelli et al. evaluated a subset of cancer patients $(n=954)$ who underwent major abdominal surgery and demonstrated that rate of VTE in patients who received fondaparinux (2.5 mg per day) was $4.7 \%$ whereas the rate of VTE in patients who received dalteparin (5000 units per day) was $7.7 \%$; the RRR was $38.6 \%$ (95\% CI: $6.7 \%$ to $59.7 \%$ ), and the incidence rate of major bleeding was $3.4 \%$ versus $2.5 \%$ $(P=0.355)$ [23]. Major bleeding occurred in $2.8 \%$ of patients who received their first fondaparinux injection at least 6 hours after surgery closure and in $3.4 \%$ of patients 
who received their first fondaparinux dose within 6 hours of surgery closure [23].

Overall, these studies suggest that fondaparinux could be an option for prevention of VTE in cancer patients who are hospitalized for either an acute medical illness or a surgical procedure.

2.4. Comparative Efficacy in VTE Treatment Trials. Primary data of fondaparinux for treatment of VTE cancer patients is also lacking. Two studies have shown the similar efficacy of fondaparinux versus LMWH and VKA for the initial phase of VTE treatment that enrolled $10 \%$ of patients with cancer $[24,25]$. A subgroup analysis of cancer patients in the Matisse-DVT trial showed that recurrent VTE rates in the initial treatment period, for the entire study period, and in patients with advanced cancer were as follows for the fondaparinux group versus the enoxaparin group: $2.4 \%$ versus $0.0 \%$, for an absolute difference of $2.4 \%(95 \% \mathrm{CI}$ : $-0.3 \%$ to $5.0 \%, P=0.080) ; 12.7 \%$ versus $5.4 \%$, for an absolute difference of $7.3 \%$ (95\% CI: $0.1 \%$ to $14.5 \%, P=$ $0.046) ; 11.5 \%$ versus $3.7 \%$, for an absolute difference of $7.8 \%$ ( $95 \%$ CI: $-6.4 \%$ to $22.0 \%, P=0.28$ ). Major bleeding rates in cancer patients during the entire study period were $7.1 \%$ in the fondaparinux group versus $7.2 \%$ in the enoxaparin group, for an absolute difference of $-0.1 \%$ (95\% CI: $-6.7 \%$ to $6.5 \%, P=0.99)$. Mortality rates were $18.3 \%$ in the fondaparinux group versus $15.3 \%$ in the enoxaparin group, for an absolute difference of $2.9 \%$ (95\% CI: $6.6 \%$ to $12.4 \%$ ). This subgroup analysis of cancer patients showed that during the entire study period there was a trend toward higher rates of recurrent VTE in fondaparinux-treated patients $(12.7 \%$ in the fondaparinux group versus $5.4 \%$ in the enoxaparin group; $P=0.046$ ) [26]. One possible explanation for the higher rates of recurrent VTE in the fondaparinux group could be that $8.7 \%$ of the fondaparinux-treated patients (bridged with VKA) had an international normalized ratio (INR) $<2.0$ (at the time of recurrent DVT versus $1.8 \%$ of the enoxaparin-treated patients; $3.6 \%$ of patients in the enoxaparin group had therapeutic INRs (2.0 to 3.0 ) versus $1.6 \%$ of patients in the fondaparinux group [27].

A subgroup analysis of cancer patients in the Matisse-PE study reported that recurrent VTEs in the initial treatment period, for the entire study period, and in patients with advanced cancer were as follows for the fondaparinux group versus the UFH group: $0.9 \%$ versus $3.9 \%$, for an absolute difference of $-3.0 \%$ ( $95 \%$ CI: $-6.8 \%$ to $0.8 \%, P=0.12$ ); $8.9 \%$ versus $17.4 \%$, for an absolute difference of $-8.3 \%$ (95\% CI: $-16.7 \%$ to $0.1 \%, P=0.054) ; 16.0 \%$ versus $29.0 \%$, for an absolute difference of $-13.0 \%$ (95\% CI: $-35.0 \%$ to $8.5 \%, P=0.24$ ), respectively. Major bleeding rates in cancer patients during the entire period were $3.6 \%$ in the fondaparinux group versus $6.3 \%$ in the UFH group, for an absolute difference of $-2.7 \%$ (95\% CI: $-8.1 \%$ to $2.7 \%$, $P=0.33$ ). Mortality rates during the 3 -month followup were $25.0 \%$ in the fondaparinux group versus $18.8 \%$ in the UFH group, for an absolute difference of $6.2 \%$ (95\% CI: $-4.2 \%$ to $16.7 \%)$. In this subgroup analysis, higher rates of recurrent VTE during the entire study were observed in the
UFH group compared with the fondaparinux group (17.2\% versus $8.9 \%, P=0.054$ ). Patients randomized to receive UFH plus VKA had lower INR rates at the time of VTE recurrence than did patients in the fondaparinux group, $7.0 \%$ and $2.7 \%$, respectively, for an absolute difference of $0.4 \%$ (95\% CI: $-10.0 \%$ to $1.0 \%)$ [27].

\subsection{Dosing of Fondaparinux}

2.5.1. Dosing of Fondaparinux in Underweight and Overweight Patients with Cancer. Fondaparinux is dosed according to the patient's body weight based on the following schema: for a body weight of $<50 \mathrm{~kg}$, the dose is $5 \mathrm{mg}$ subcutaneously (SQ) daily; for a body weight of $50 \mathrm{~kg}$ to $100 \mathrm{~kg}$, the dose is $7.5 \mathrm{mg}$ SQ daily; for a body weight $>100 \mathrm{~kg}$, the dose is $10 \mathrm{mg}$ SQ daily [18].

Pooled analysis of the effect of obesity (cutoff, $30 \mathrm{~kg} / \mathrm{m}^{2}$ ) on outcomes in the Matisse trials (Matisse-DVT and Matisse$\mathrm{PE})$ has been reported. Body weight (kg) and body mass index $\left(\mathrm{BMI} ; \mathrm{kg} / \mathrm{m}^{2}\right)$ ranges reported in this analysis were from $33 \mathrm{~kg}$ to $216 \mathrm{~kg}$ and from $12.8 \mathrm{~kg} / \mathrm{m}^{2}$ to $8.1 \mathrm{~kg} / \mathrm{m}^{2}$, respectively [28]. When investigators analyzed the data from the Matisse trials, they observed no difference in recurrent VTE rates between fondaparinux and heparins (enoxaparin or UFH) across body weight and BMI categories as follows: body weight $\leq 100 \mathrm{~kg}$ ( $3.90 \%$ versus $4.45 \%, P=0.42)$; body weight $>100 \mathrm{~kg}(4.0 \%$ versus $5.7 \%, P=0.41)$; BMI $<30 \mathrm{~kg} / \mathrm{m}^{2}$ (3.9\% versus $\left.4.5 \%, P=0.42\right) ;$ BMI $\geq 30 \mathrm{~kg} / \mathrm{m}^{2}$ (3.7\% versus $4.8 \%, P=0.40)$. Bleeding rates did not differ between fondaparinux and heparins (enoxaparin or UFH): body weight $\leq 100 \mathrm{~kg}$ ( $1.3 \%$ versus $1.2 \%, P=0.77)$; body weight $>100 \mathrm{~kg}(0.4 \%$ versus $0.8 \%, P=0.62)$; BMI $<30 \mathrm{~kg} / \mathrm{m}^{2}(1.5 \%$ versus $1.2 \%, P=0.53) ; \mathrm{BMI} \geq 30 \mathrm{~kg} / \mathrm{m}^{2}$ $(0.3 \%$ versus $1.1 \%)$ [28]. The results of this study showed that the safety and efficacy of fondaparinux for initial treatment of VTE was comparable to heparin across different body weight and BMI categories [28]. In the Matisse trials, cancer patients comprised $25 \%$ of total patients enrolled overall $[24,25]$. Until further evidence is available in cancer patients with extremes of body weight and BMI, data presented by the pool analysis should be interpreted with caution.

2.5.2. Dosing of Fondaparinux in Cancer Patients with Renal Disease. Fondaparinux is excreted via the kidneys, is contraindicated in patients with $\mathrm{CrCl}<30 \mathrm{~mL} / \mathrm{min}$, and should be used with caution in patients with $\mathrm{CrCl}$ from 30 to $50 \mathrm{~mL} / \mathrm{min}$ [18]. Close attention to estimation of the glomerular filtration rate (GFR) is imperative in dosing of antithrombotics such as fondaparinux that has a long $t_{1 / 2}$.

Estimation of the GFR calculated with the CockcroftGault (CG) formula differs widely from calculations made with the Modification of Diet in Renal Disease (MDRD) formula in low BMIs and in patients older than 75 years [29]. Another study reported higher rates of bleeding and transfusions in patient with acute coronary syndrome that were prescribed unadjusted doses of antithrombotics based on CG [30]. 
The CG formula may better assess GFR in patients with normal serum creatinine (Scr) levels who are at risk of developing kidney disease such as patients who have diabetes, who have stage 1 or 2 CKD, or who are elderly. The MDRD formula may be more appropriate for predicting the GFR in patients with CKD who do not have normal Scr levels, who have a GFR $<30 \mathrm{~mL} / \mathrm{min}$, and who have a high BMI. Neither CG nor MDRD was accurate in the following patients: heart transplant patients, kidney donors, patients with advanced liver disease, and hospitalized patients [26].

In a pharmacokinetic simulation study, Turpie et al. showed that dosing fondaparinux at $1.5 \mathrm{mg}$ and $2.5 \mathrm{mg}$ SQ daily provided comparable trough plasma concentration $\left(C_{\min }\right)$, peak plasma concentration $\left(C_{\max }\right)$, and area under the curve $\left(\mathrm{AUC}_{0-24 \mathrm{~h}}\right)$ at days 1,7 , and 28 in patients with a $\mathrm{CrCl}$ of $\geq 20 \mathrm{~mL} / \mathrm{min},<50 \mathrm{~mL} / \mathrm{min}$, and $\mathrm{CrCl}>50 \mathrm{~mL} / \mathrm{min}$. However, dosing fondaparinux at $2.5 \mathrm{mg}$ produced much higher $C_{\min }, C_{\max }$, and $\mathrm{AUC}_{0-24 \mathrm{~h}}$ in patients with $20 \mathrm{~mL} / \mathrm{min}$ $\geq \mathrm{CrCl}<50 \mathrm{~mL} / \mathrm{min}$ [31]. The fondaparinux 1.5-mg dosing schedule may be a safe and effective alternative to $2.5-\mathrm{mg}$ recommended dose for the prevention of VTE; however, determining the most effective dosing schedule for fondaparinux requires further trials and investigation.

Results from studies of fondaparinux $(2.5 \mathrm{mg})$ used as an anticoagulant during hemodialysis have shown the efficacy of fondaparinux in preventing circuit clotting during dialysis; however, anti-Xa activity is increased and may potentially increase the risk of bleeding in such patients on dialysis $[32,33]$.

To our knowledge, there have been no studies assessing the safety and efficacy of fondaparinux in patients with endstage renal disease and concomitant VTE. Dialysis patients with an acute medical illness who are hospitalized should receive prophylaxis with $\mathrm{UFH}[34]$.

2.6. Monitoring Anti-Xa Levels in Patients Receiving Fondaparinux. Currently, to our knowledge, there have been no clinical trials to validate peaks and troughs in the efficacy of fondaparinux.

Fondaparinux-specific anti-Xa assay should be used to monitor the anti-Xa levels of fondaparinux in patients who have unstable renal function, are elderly, are morbidly obese, or have a low BMI [35]. Activated partial thromboplastin time (aPTT) and prothrombin time (PT) cannot be used to measure anticoagulant properties of fondaparinux because these test systems measure the inhibition of thrombin and not factor Xa [36]. Fondaparinux has been shown to affect the protein $S$ assay in vitro, resulting in false elevation of protein S levels; however, the significance of these findings is yet to be determined [37]. Linkins et al. have shown that fondaparinux has no effect on aPTT or activated clotting time (ACT), contrary to heparins; therefore, such tests are insensitive to the anticoagulant properties of fondaparinux [38]. Smogorzewska et al. showed that fondaparinux (prophylactic or therapeutic dose) prolonged PT by 1 second and the aPTT by 4 to 5 seconds without significant changes in fibrinogen, AT, and thrombin time assays [39]. Depasse et al. showed that LMWH anti-Xa (IU/mL) levels varied significantly between different assays for the same fondaparinux concentrations $(P<0.01)[40]$. Methodology of determining factor Xa activity of fondaparinux requires a chromogenic assay that is calibrated to fondaparinux but not to $\mathrm{LMWH}$ or UFH.

2.7. The Role of Fondaparinux in Critically Ill Cancer Patients. Despite prophylaxis use in the intensive care unit (ICU), the rate of DVT is about $5.1 \%$ to $5.8 \%$ and the rate of $\mathrm{PE}$ is about $1.2 \%$ to $2.3 \%$, the major bleeding rate is approximately $13.0 \%$, and the mortality rate is $15.0 \%$ [41]. The pharmacokinetic properties of drugs in ICU patients may be altered owing to reduced hepatic metabolism, reduced transport function, intestinal atrophy, increased or decreased extravascular fluids, reduced $\mathrm{CrCl}$, altered tissue permeability, or decreased protein binding of the drugs [42].

Administration of vasopressors to patients who received fondaparinux $(2.5 \mathrm{mg})$ did not change the mean range of anti-Xa levels compared with those without vasopressors ( 0.2 to $0.4 \mathrm{IU} / \mathrm{mL}$ versus 0.3 to $0.2 \mathrm{IU} / \mathrm{mL}, P=0.06)$. Interestingly, investigators found no occurrence VTE and 2 minor bleeds with anti-Xa of 0.24 and $0.44 \mathrm{IU} / \mathrm{mL}$ [43].

There are currently no guidelines for dose adjustment of fondaparinux based on anti-Xa levels, and, to our knowledge, no studies have compared the effectiveness of fondaparinux versus heparin in ICU patients. Use of pharmacological agents for DVT prophylaxis in ICU patients should be based on evidence, the half-life of the agent, and the availability of an antidote if bleeding occurs.

2.8. Role of Fondaparinux in Cancer Patients with HeparinInduced Thrombocytopenia. Heparin-induced thrombocytopenia (HIT) is an immune-mediated disorder caused by immunoglobulin $\mathrm{G}$ (IgG) antibodies that bind to platelet factor 4 (PF4), resulting in the generation and release of procoagulant microparticles such as thrombin [44]. Thrombin causes disseminated arterial and venous thrombi and has a reported absolute risk of thrombosis of $35 \%$ to $57 \%$ in some clinical settings [45]. Mortality rates in patients with HIT and thrombosis can be as high as 30\%, and $20 \%$ of patients who survive require a limb amputation [46].

Patients who can carry risk of HIT of $>1 \%$ are surgical patients who received UFH (prophylactic dose or treatment dose) for more than 4 days. Patients who carry risk of HIT of $0.1 \%$ to $1 \%$ are (1) medical patients who received UFH (prophylactic dose or therapeutic dose) for more than 4 days; (2) surgical patients who received LMWH for more than 4 days or heparin flushes for more than 4 days; (3) medical or surgical patients who were switched from UFH to LMWH carry an estimated risk HIT of $0.1 \%$ to $1.0 \%$. Finally, medical or surgical patients who received LMWH for more than 4 days, medical patients received heparin flushes only, or any patients that have who received UFH or LMWH for fewer than 4 days have a risk of HIT of $0.1 \%$ [47].

Fondaparinux is considered a pentasaccharide and has an average molecular weight of $1.7 \mathrm{kDa}$ and only 5 saccharide residue. Evidence suggests that an increased risk for HITrelated antigen production is dependent on the molecular 
weight and length of the polysaccharides $(>2.4 \mathrm{kDa}$ and $>10$ saccharide units). Typically, in LMWHs, the range of molecular weight and saccharide residues vary from $4.5 \mathrm{kDa}$ to $7.5 \mathrm{kDa}$ and 13 to 15 saccharide residues, respectively. In UFH, the molecular weight and saccharide residues are much higher, $15 \mathrm{kDa}$ and $45 \mathrm{kDa}$. It has been hypothesized that the lower saccharide residue, the lower antigenicity [48].

Testing the sera of patients from 2 clinical trials, investigators showed that the PF4-fondaparinux complex, but not the PF4-LMWH complex, is readily recognized by antibodies generated during HIT and that the risk of HIT associated with fondaparinux is very low [49]. Kovacs reported a successful case series of five patients with the diagnosis of HIT who were treated with $7.5 \mathrm{mg}$ of fondaparinux [50]. In this case series, time to platelet recovery was between 2 and 9 days, with no new thrombotic activity or major bleeding [50].

Wester et al., in a retrospective study of seven critically ill patients with suspected HIT, investigated the role of fondaparinux at a dosing schedule of $2.5 \mathrm{mg}$ per day, with dose adjustments according to renal function (adjusted to $1.5 \mathrm{mg}$ ) [51]. HIT antibodies were absent in all patients; five patients were classified as unlikely to have HIT, whereas two patients were classified as possibly having HIT. During treatment with fondaparinux, platelets recovered in five patients but did not recover in the other two. The authors concluded that the role of fondaparinux in cases of suspected HIT might be as bridging until HIT is confirmed [51].

Several case reports in the literature have advocated the use of a therapeutic dose of fondaparinux in the management of HIT [52]. Lobo et al. showed in a prospective pilot trial comparing HIT patients treated with fondaparinux $(n=$ 7) with historical controls treated with direct thrombin inhibitor (DTI; $n=10$ ) that 7 of 7 fondaparinux-treated patients had complete platelet recovery time (increase from baseline by at least $30 \%$ of nadir to $>100,000 / \mathrm{mm}^{3}$ by day 7) and no fondaparinux-treated patients experienced new thrombotic events, major bleeding, or death by day 30 . In the historical control group, 8 of 10 patients achieved platelet recovery with no new thrombotic events or major bleeding, but 4 had limb gangrene that might have been due to inappropriate dosing of warfarin in relation to the diagnosis of HIT [53].

NCCN guidelines considers use of fondaparinux for treatment of HIT as unlabeled use, and the ACCP suggests that after recovery of platelet levels during initial treatment with DTI, the DTI can be replaced with fondaparinux in therapeutic doses and bridging to warfarin $[5,47]$.

The use of fondaparinux in patients with HIT has been challenged. In 2003, Warkentin et al. reported a platelet serotonin-release assay of $90 \%$ (normal value, <20\%) and an anti-PF4 polyanion enzyme immunoassay of 1.871 units (normal value, $<0.40$ ) in a patient who was not predisposed to heparin and was postoperatively on fondaparinux (2.5mg dosing schedule), with a concomitant platelet drop and signs of thrombosis. The authors indicated that in rare cases fondaparinux can activate antibodies against PF4 that resemble the antibodies in HIT [54].
Rota et al. reported a strong optical density (OD) of 1.700 units without thrombosis in a patient exposed to fondaparinux after undergoing a total hip replacement. Three years earlier, the patient had a history of HIT with thrombosis due to nadroparin. The authors concluded that fondaparinux should be used with caution in patients with HIT [55].

Recently, Alsaleh et al. also reported an OD of 1.75 units (normal value, $<0.45 \mathrm{U}$ ), heparin-dependent platelet activation at a dilution of 1 in $8(3 \%$ release at $0 \mathrm{U} / \mathrm{mL}$, $100 \%$ at $0.1 \mathrm{U} / \mathrm{mL}$, and $0 \%$ at $100 \mathrm{U} / \mathrm{mL} \mathrm{UFH}$ ), with new DVT and concomitant thrombocytopenia in a patient on fondaparinux prophylaxis following a surgical procedure. The patient had been on prophylaxis with LMWH postoperatively for 3 days. In this case, platelet activation was also observed with pharmacological doses of enoxaparin $(0.1 \mathrm{U} / \mathrm{mL})$, but not in the presence of fondaparinux [56]. Treatment of cancer patients with HIT should not differ from treatment of patients who do not have cancer, and treatment consists of DTIs (such as lepirudin, bivalirudin, or argatroban) for the acute phases of HIT followed by longterm VKA after platelet recovery [57].

Until further evidence is available from randomized clinical trials, fondaparinux should be used with caution to treat HIT in cancer patients.

2.9. Bleeding Risk Assessment. To date, there is no validated bleeding risk assessment in cancer patients; however, several bleeding assessment tools have been examined in clinical trials. Assessment of bleeding risk will enable closer monitoring of patients who are at risk of bleeding while on anticoagulation therapy. In one study, risk of bleeding associated with anticoagulation in cancer patients was about $4.2 \%$ during 90 days of therapy, with a mortality rate greater than $60 \%$ within 30 days of a major bleeding event [58].

Data for management of VTE in cancer patients with thrombocytopenia is lacking. Transient thrombocytopenia due to chemotherapy doubles the bleeding risk from $10 \%$ at a platelet count of $20,000 / \mathrm{mm}^{3}$ to $20 \%$ when the platelet count drops below $10,000 / \mathrm{mm}^{3}$ in solid tumor patients [59].

NCCN considers platelet counts of less than $50,000 / \mathrm{mm}^{3}$ as a contraindication to prophylactic or therapeutic anticoagulation therapy $[5,6]$. ASCO recommends using therapeutic anticoagulation in cancer patients with preexisting thrombocytopenia with caution. Validated bleeding assessment tools in the literature are evolving. The prospective registry Registro Informatizado de La Enfermedad Thromboembólicà (RIETE) investigators have identified variables such as recent major bleed, renal dysfunction, anemia, cancer, clinically overt PE, and advanced age as being associated with major bleeding. When RIETE investigators assigned point scores for bleeding based on risk factors, the incident rate of major bleeding was $0.1 \%$ for low risk, $2.8 \%$ for intermediate risk, and $6.2 \%$ for high-risk patients. (Table 3) [60].

In a recent analysis of the prospective RIETE registry, investigators identified variables such as age $>75$ years, recent major bleeding, immobility $\geq 4$ days, metastatic cancer, anemia, platelet count $<100,000 / \mathrm{mm}^{3}$, abnormal PT, and 
TABLe 3: Multivariate analysis for major bleeding and bleeding risk index classification $[60,61]$.

(a)

\begin{tabular}{lccc}
\hline Risk factors & Odds ratio $(95 \%$ CI) & $P$ value & Points \\
\hline Recent major bleed & $2.7(1.6-4.6)$ & $<0.001$ & 2.0 \\
Serum creatinine $(\mathrm{Scr})>1.2 \mathrm{mg} / \mathrm{dL}$ & $2.1(1.7-2.8)$ & $<0.001$ & 1.5 \\
Anemia & $2.1(1.7-2.7)$ & $<0.001$ & 1.5 \\
Cancer & $1.7(1.4-2.2)$ & $<0.001$ & 1.0 \\
Clinically overt pulmonary embolism & $1.7(1.4-2.2)$ & $<0.001$ & 1.0 \\
Age $>75$ years & $1.7(1.3-2.1)$ & $<0.001$ & 1.0 \\
\hline
\end{tabular}

0 points $=$ low risk.

$0.1 \%$ (95\% CI: $0.0-0.2$ ).

$1-4$ points $=$ intermediate risk.

$2.8 \%$ (95\% CI: $2.4-3.3$ ).

$>4$ points $=$ high risk.

$7.3 \%$ (95\% CI: 4.0-9.1).

(b)

\begin{tabular}{lccc}
\hline Risk factors & Odds ratio $(95 \% \mathrm{CI})$ & $P$ value & Points \\
\hline Age $>75$ years & $2.16(1.49-3.16)$ & $<0.001$ & 0.002 \\
Recent major bleed & $2.64(1.44-4.83)$ & $<0.001$ & 1.0 \\
Immobility $\geq 4$ days & $1.99(1.40-2.83)$ & $<0.001$ & 1.0 \\
Metastatic cancer & $3.80(2.56-5.64)$ & 0.021 & 2.0 \\
Anemia & $1.54(1.07-2.22)$ & 0.016 & 1.0 \\
Platelet count $<100.000 / \mathrm{mm}^{3}$ & $2.23(1.16-4.29)$ & 0.001 & 1.0 \\
Elevated prothrombin time $(\mathrm{PT})$ & $2.09(1.34-3.26)$ & $<0.001$ & 1.0 \\
Creatinine clearance $(\mathrm{CrCl})<30 \mathrm{~mL} / \mathrm{min}$ & $2.27(1.49-3.44)$ & 0.038 & 1.0 \\
Distal deep vein thrombosis & $0.39(0.16-0.95)$ & & -1.0 \\
\hline
\end{tabular}

Score $<1.5=$ low risk $0.16 \%$.

LR $=0.29$ (95\% CI: 0.20-0.41).

Score $1.5-4=$ intermediate risk $1.06 \%$.

LR $=1.92$ (95\% CI: 1.69-2.17).

Score $>4=$ high risk $4.24 \%$.

$\mathrm{LR}=7.95$ (95\% CI: 5.42-11.6).

$\mathrm{CrCl}<30 \mathrm{~mL} / \mathrm{min}$ as independent risk factors for bleeding. Then investigators composed a risk scoring system based on assigned points to each variable to predict the risk of fatal bleeding during 90 days following diagnosis of VTE. Patients with a risk score of $<1.5$ points had a bleeding incidence rate of $0.16 \%$, those with a risk score of 1.5 to 4.0 had a bleeding incidence of $1.06 \%$, and those with score of $>4.0$ had a incidence of $4.24 \%$ of fatal bleeding with the likelihood ratio 0.29 for the low-risk group, 1.92 for the moderaterisk group, and 7.95 for the high-risk group (Table 3) [61]. We recommend that patients with any of the following risk factors for major bleeding should be closely monitored while on anticoagulation: age ( $>75$ years), presence of metastatic cancer, immobility, platelet count $<100,000 / \mathrm{mm}^{3}$, anemia, recent major bleed, coagulopathy, and $\mathrm{CrCl}<30 \mathrm{~mL} / \mathrm{min}$.

2.10. Reversal of Fondaparinux. As described before, the incidence of bleeding associated with fondaparinux ranges from $1.1 \%$ to $7.2 \%[27,28]$.

Following the assessment of bleeding risk, choosing long-acting anticoagulants requires adequate reversibility by an antidote in cases of bleeding. VKAs can be reversed with administration of vitamin K, LMWH can be partially reversed with the administration of protamine, and UFH can be completely reversed with administration of protamine; in bleeding events, transfusion of red blood cells is required as well [62].

There is limited experience with reversal of fondaparinux in bleeding cases, and, to date, there are no studies conducted specifically in cancer patients. There are case reports and limited laboratory studies alluding to judicious use of recombinant factor VIIa (rVIIa) for reversal of bleeding associated with fondaparinux. rVIIa is an activated factor VIIa that is approved for use in cases of congenital factor VII deficiency, patients with factor VIII or IX inhibitors, and cases of Glanzmann thrombasthenia; however, in recent years, it has been used "off-label" for reversal of warfarin, central nervous system bleeding, uremia, bone marrow transplantation, type III von Willebrand disease, factor XI deficiency, Jehovah's Witness hematologic malignancies, liver disease (cirrhosis, liver transplantation, or fulminant hepatic failure), and in platelet disorders (quantitative and qualitative). rVIIa primarily binds to TF following injury to the vessel wall and activates factor $\mathrm{X}$ to factor $\mathrm{Xa}$, leading to conversion of prothrombin to thrombin. Thrombin activates platelets, converts factor V to factor Va and factor VIII to factor VIIIa, 
and finally activates thrombin-activable fibrinolysis inhibitor (TAFI), which downregulates fibrinolysis. The usual dose if rVIIa is $90 \mu \mathrm{g} / \mathrm{kg}$ administered intravenously [63].

In a randomized, placebo-controlled study of 16 healthy male subjects who received a single dose of fondaparinux $(10 \mathrm{mg})$, the effect of a single dose of $90 \mu / \mathrm{kg}$ intravenous (IV) bolus of rFVIIa was studied on coagulation parameters. Thrombin generation time (TGT) was rapidly normalized by administration of rFVIIa and persisted for 6 hours $(P<0.001)$. The endogenous thrombin potential (ETP) at 2 and 8 hours was $9 \%$ higher in the fondaparinux plus rVIIa than in the fondaparinux group alone $(P=0.056)$. Prothrombin activation was increased by $34 \%$ at 2 and 8 hours in the fondaparinux plus rVIIa compared with the fondaparinux group alone $(P=0.022)$. The AUC analysis between time points 2 and 8 hours showed a significant reduction in the aPTT $(P=0.015)$. The PT was reduced $26 \%$ after the administration of rVIIa (14.3 \pm 0.9 to $9.2 \pm$ 0.9 seconds; $P<0.0001)$. The authors concluded that administration of rFVIIa as a single $90 \mu / \mathrm{kg}$ IV bolus could neutralize the anticoagulant effect of fondaparinux $(10 \mathrm{mg})$ [64].

The effect of $90 \mu / \mathrm{kg}$ IV bolus of rVIIa on clot lysis time in 8 healthy volunteers when added to $10 \mathrm{mg}$ of fondaparinux was also studied by Lisman et al. In this study, the authors found a decrease in the clot lysis time on addition of fondaparinux (control $80 \pm 13$ minutes, fondaparinux $65 \pm$ 15 minutes; mean $\pm \mathrm{SD}, P<0.001$ ), and on addition of rVIIa to fondaparinux-anticoagulated plasma in this group, a significant increase in clot lysis time was observed (from $65 \pm 15$ minutes to $72 \pm 13$ minutes, $P<0.01$ ), and this effect lasted for up to 8 hours. The authors concluded that rVIIa may be an alternative option for reversal of bleeding due to fondaparinux [65].

Gerotziafas and his colleagues have concluded that recombinant factor VII (rFVIIa) partially reverses the effect of fondaparinux on inhibition of thrombin generation. In their study, the effect of concentrations of fondaparinux from 0.1 to $1.0 \mu \mathrm{g} / \mathrm{mL}$ (concentrations required for prophylaxis and treatment of VTE) and the influence of rVIIa at $1 \mu \mathrm{g} / \mathrm{mL}$ on various parameters such as lag time (minutes), thrombin $C_{\max }(\mathrm{nM}), T_{\max }$ (minutes), and ETP $(\mathrm{nM} \times$ minutes) were studied. In the presence of fondaparinux $0.5 \mu \mathrm{g} / \mathrm{mL}$ rVIIa, both the lag time and $T_{\max }$ of thrombin generation were decreased by 1 minute. At $0.8 \mu \mathrm{g} / \mathrm{mL}$ to $1.0 \mu \mathrm{g} / \mathrm{mL}$ of fondaparinux, rVIIa reduced the lag time by $3 \pm 1.5$ minutes and the $T_{\max }$ of thrombin generation by $4 \pm 2$ minutes $(P<0.05)$. The addition of rVIIa to $0.5 \mu \mathrm{g} / \mathrm{mL}$ or lower concentration of fondaparinux did not modify the $C_{\max }$ and ETP, but at concentrations of $0.8 \mu \mathrm{g} / \mathrm{mL}$ of fondaparinux, rVIIa slightly increased $C_{\max }$ and ETP $(63 \pm 15 \mathrm{nM})$ and (840 $\pm 330 \mathrm{nM} \times$ minutes $)(P>0.05)[66]$.

A case of rVIIa $(90 \mu \mathrm{g} / \mathrm{kg})$ to a 76 -year-old patient who was on fondaparinux $(7.5 \mathrm{mg})$ following head trauma due to falls has been described in the literature. The patient received the dose of rVIIa prior to craniotomy for evacuation of a cranial hematoma; however, the patient did not survive the trauma due to increasing intracranial pressure following craniotomy [67].
In another case report of posthip replacement bleeding following fondaparinux use, rVIIa $(90 \mu \mathrm{g} / \mathrm{kg})$, tranexamic acid $(15 \mathrm{mg} / \mathrm{kg})$, and transfusion of packed red blood cells successfully stabilized hemoglobin levels [68]. A recent metaanalysis reported the safety of rVIIa in randomized clinical trials. Levi et al. reported higher rates of arterial events (mostly acute coronary syndrome) in patients who received rVIIa than in patients who received a placebo (5.5\% versus $3.2 \%$; OR: $1.68,95 \%$ CI: $1.20 \%$ to $2.36 \%, P=0.003)$. This difference was significant in patients $\geq 65$ years $(9 \%$ versus $3.8 \%$; OR: $2.43,95 \% \mathrm{CI}: 1.34 \%$ to $4.41 \%, P=$ 0.003). The dose of rVIIa ( 80 to $120 \mu \mathrm{g} / \mathrm{kg}$ ) was also a significant covariate for arterial embolic events in patients who received rVIIa for central nervous system bleeding $(P=$ 0.02 ) [69]. The cost of rVIIa use in treatment of minor-tomoderate bleeding events in patients with hemophilia has been reported to be $\$ 30,818.00$ [70]. The use of rVIIa in bleeding episodes associated with fondaparinux should be done with caution given the cost, risk of thrombotic events, and partial reversibility.

\section{Idraparinux}

Idraparinux, another long-acting factor Xa inhibitor $(2.5 \mathrm{mg}$ SQ weekly dose), currently is being investigated in clinical trial for the prevention and treatment of VTE. Idraparinux has a longer $t_{1 / 2}$ (up to 60 days) and has no antidote (Table 2) $[17,18]$. The van Gogh trial showed noninferiority of idraparinux versus standard of care (UFH with VKA) for VTE treatment (2.9\% versus 3.0\%;OR: 0.98, 95\% CI: $0.63 \%$ to $1.50 \%$ ). Major bleeding events in the idraparinux group versus standard-of-care group were $4.5 \%$ versus $7.0 \%$ $(P=0.004)$. In the PE arm of the study, the rate of recurrent $\mathrm{PE}$ in the idraparinux versus standard of care was $3.5 \%$ versus $1.6 \%$ (OR: $2.14,95 \%$ CI: $1.21 \%$ to $3.78 \%)$, which did not meet the noninferiority criteria [71]. Recurrent VTE in six-month subgroup analysis of cancer patients enrolled in of the van Gogh trial was $2.5 \%$ in the idraparinux group compared to $6.4 \%$ in the standard of care group (hazard ration 0.39 , 95\%CI: $0.14-1.11$ ). The rate of bleeding was comparable (OR 0.89, 95\% CI: 0.50-1.59) [72]. These results need further investigation in forthcoming idraparinux trials. Currently, one case report of successful reversal of idraparinux with rVIIa $(30 \mu \mathrm{g} / \mathrm{kg})$ exists in the literature [73]. The role of idraparinux in cancer patients undergoing surgical procedures also needs to be investigated. Shorter acting anticoagulants such as heparins should be used in surgical settings where epidurals are indicated.

\section{Idrabiotaparinux}

Idrabiotaparinux is another long-acting factor Xa inhibitor (3.0 mg SQ weekly dose) that has a $t_{1 / 2}$ of up to 60 days and has no antidote (Table 2). Addition of a biotin moiety to the molecule of idraparinux has been shown in animal studies to be neutralized by avidin (egg protein), an antidote in bleeding cases. A recent study of treatment of VTE (cancer patients comprised $\sim 5 \%$ ) comparing idrabiotaparinux 
(3.0 mg SQ weekly) versus idraparinux (2.5 mg SQ weekly) for 180 days showed equal factor $\mathrm{Xa}$ inhibition, recurrent VTE of $2.3 \%$ versus $3.2 \%$ (difference of $-0.9 \%$, 95\% CI: $-3.2 \%$ to $-1.4 \%$ ), clinically relevant bleeding of $5.2 \%$ versus $7.3 \%$ (difference of $-2.1 \%, 95 \% \mathrm{CI}:-5.6 \%$ to $-1.4 \%, P=$ 0.29 ), and a mortality rate of $1.6 \%$ versus $3.2 \%$ (difference of $1.7 \%, 95 \%$ CI: $-3.9 \%$ to $-0.5 \%)$. Three patients in the idrabiotaparinux group required avidin for planned procedures with favorable outcomes [74]. Overall results showed the comparative efficacy and safety of idrabiotaparinux to idraparinux. Further trials are needed to assess safety and efficacy of this agent in cancer patients.

\section{Conclusion}

More targeted anticoagulants such as factor Xa inhibitors are being investigated. To date, fondaparinux has received FDA approval for the prevention and treatment of VTE. Such long-acting agents provide feasibility and better compliance than VKA or heparins. Evidence suggests that fondaparinux should be used in the acute phase of VTE treatment, but evidence of the efficacy and safety for long-term treatment is lacking, especially in cancer patients. Monitoring patients for bleeding, especially those at high risk for bleeding, is necessary owing to the fact that long-acting agent reversal is partially possible. Evidence of the newer, longer acting factor $\mathrm{Xa}$ inhibitors such as idraparinux and idrabiotaparinux is clearly lacking at present.

\section{Conflict of Interests}

The authors do not report any conflict of interests regarding this work.

\section{Acknowledgment}

The authors would like to thank Sue Moreau from the Department of Scientific Publications at The University of Texas MD Anderson Cancer Center for editing this paper.

\section{References}

[1] A. Varki, "Trousseau's syndrome: multiple definitions and multiple mechanisms," Blood, vol. 110, no. 6, pp. 1723-1729, 2007.

[2] D. Bergqvist, "Risk of venous thromboembolism in patients undergoing cancer surgery and options for thromboprophylaxis," Journal of Surgical Oncology, vol. 95, no. 2, pp. 167-174, 2007.

[3] A. Stanley and A. Young, "Primary prevention of venous thromboembolism in medical and surgical oncology patients," British Journal of Cancer, vol. 102, no. 1, pp. S10-S16, 2010.

[4] A. A. Khorana, C. W. Francis, E. Culakova, N. M. Kuderer, and G. H. Lyman, "Frequency, risk factors, and trends for venous thromboembolism among hospitalized cancer patients," Cancer, vol. 110, no. 10, pp. 2339-2346, 2007.

[5] National Comprehensive Cancer Network Clinical Practice in Oncology, "Venous thromboembolic disease," 2010, http://www.nccn.org/.
[6] G. H. Lyman, A. A. Khorana, A. Falanga et al., "American Society of Clinical Oncology Guideline: recommendations for venous thromboembolism prophylaxis and treatment in patients with cancer," Journal of Clinical Oncology, vol. 25, no. 34, pp. 5490-5505, 2007.

[7] W. H. Geerts, D. Bergqvist, G. F. Pineo et al., "Prevention of venous thromboembolism: American College of Chest Physicians evidence-based clinical practice guidelines (8th edition)," Chest, vol. 133, no. 6, pp. 381S-453S, 2008.

[8] C. Kearon, S. R. Kahn, G. Agnelli, S. Goldhaber, G. E. Raskob, and A. J. Comerota, "Antithrombotic therapy for venous thromboembolic disease: American College of Chest Physicians evidence-based clinical practice guidelines (8th edition)," Chest, vol. 133, no. 6, pp. 454S-545S, 2008.

[9] B. Saraiya and S. Goodin, "Management of venous thromboembolism and the potential to impact overall survival in patients with cancer," Pharmacotherapy, vol. 29, no. 11, pp. 1344-1356, 2009.

[10] E. A. Akl, G. Kamath, V. Yosuico et al., "Thromboprophylaxis for patients with cancer and central venous catheters," Cancer, vol. 112, no. 11, pp. 2483-2492, 2008.

[11] P. L. Gross and J. I. Weitz, "New antithrombotic drugs," Clinical Pharmacology and Therapeutics, vol. 86, no. 2, pp. 139-146, 2009.

[12] M. N. Levine, "New antithrombotic drugs: potential for use in oncology," Journal of Clinical Oncology, vol. 27, no. 29, pp. 4912-4918, 2009.

[13] J. M. Walenga, W. P. Jeske, M. M. Samama, F. X. Frapaise, R. L. Bick, and J. Fareed, "Fondaparinux: a synthetic heparin pentasaccharide as a new antithrombotic agent," Expert Opinion on Investigational Drugs, vol. 11, no. 3, pp. 397-407, 2002.

[14] A. G. G. Turpie, "Fondaparinux: a factor Xa inhibitor for antithrombotic therapy," Expert Opinion on Pharmacotherapy, vol. 5, no. 6, pp. 1373-1384, 2004.

[15] N. A. Reynolds, C. M. Perry, and L. J. Scott, "Fondaparinux sodium: a review of its use in the prevention of venous thromboembolism following major orthopaedic surgery," Drugs, vol. 64, no. 14, pp. 1575-1596, 2004.

[16] H. J. Rupprecht and R. Blank, "Clinical pharmacology of direct and indirect factor xa inhibitors," Drugs, vol. 70, no. 16, pp. 2153-2170, 2010.

[17] J. Harenberg, "Development of idraparinux and idrabiotaparinux for anticoagulant therapy," Thrombosis and Haemostasis, vol. 102, no. 5, pp. 811-815, 2009.

[18] GlaxoSmithKline Inc, Arixtra (fondaparinux) Prescribing Information, GlaxoSmithKline, Research Triangle Park, NC, USA, 2010.

[19] I. Ahrens, G. Y. H. Lip, and K. Peter, "New oral anticoagulant drugs in cardiovascular disease," Thrombosis and Haemostasis, vol. 104, no. 1, pp. 49-60, 2010.

[20] F. Donat, J. P. Duret, A. Santoni et al., "The pharmacokinetics of fondaparinux sodium in healthy volunteers," Clinical Pharmacokinetics, vol. 41, no. 2, pp. 1-9, 2002.

[21] A. T. Cohen, B. L. Davidson, A. S. Gallus et al., "Efficacy and safety of fondaparinux for the prevention of venous thromboembolism in older acute medical patients: randomised placebo controlled trial," British Medical Journal, vol. 332, no. 7537, pp. 325-327, 2006.

[22] A. G. G. Turpie, K. A. Bauer, J. A. Caprini, P. C. Comp, M. Gent, and J. E. Muntz, "Fondaparinux combined with intermittent pneumatic compression vs. intermittent pneumatic compression alone for prevention of venous thromboembolism after abdominal surgery: a randomized, doubleblind comparison," Journal of Thrombosis and Haemostasis, vol. 5, no. 9, pp. 1854-1861, 2007. 
[23] G. Agnelli, D. Bergqvist, A. T. Cohen, A. S. Callus, and M. Gent, "Randomized clinical trial of postoperative fondaparinux versus perioperative dalteparin for prevention of venous thromboembolism in high-risk abdominal surgery," British Journal of Surgery, vol. 92, no. 10, pp. 1212-1220, 2005.

[24] H. R. Büller, B. L. Davidson, H. Decousus et al., "Subcutaneous Fondaparinux versus Intravenous Unfractionated Heparin in the Initial Treatment of Pulmonary Embolism," The New England Journal of Medicine, vol. 349, no. 18, pp. 1695-1702, 2003.

[25] H. R. Büller, B. L. Davidson, H. Decousus et al., "Fondaparinux or enoxaparin for the initial treatment of symptomatic deep vienous thrombosis: a randomized trial," Annals of Internal Medicine, vol. 140, no. 11, 2004.

[26] R. Helou, "Should we continue to use the Cockcroft-Gault formula?" Nephron-Clinical Practice, vol. 116, no. 3, pp. c172-c185, 2010.

[27] F. F. van Doormaal, G. E. Raskob, B. L. Davidson et al., "Treatment of venous thromboembolism in patients with cancer: subgroup analysis of the Matisse clinical trials," Thrombosis and Haemostasis, vol. 101, no. 4, pp. 762-769, 2009.

[28] B. L. Davidson, H. R. Büller, H. Decousus et al., "Effect of obesity on outcomes after fondaparinux, enoxaparin, or heparin treatment for acute venous thromboembolism in the Matisse trials," Journal of Thrombosis and Haemostasis, vol. 5, no. 6, pp. 1191-1194, 2007.

[29] I. Gouin-Thibault, E. Pautas, I. Mahé et al., "Is modification of diet in renal disease formula similar to Cockcroft-Gault formula to assess renal function in elderly hospitalized patients treated with low-molecular-weight heparin?" Journals of Gerontology-Series A, vol. 62, no. 11, pp. 1300-1305, 2007.

[30] C. Melloni, E. D. Peterson, A. Y. Chen et al., "Cockcroft-gault versus modification of diet in renal disease. Importance of glomerular filtration rate formula for classification of chronic kidney disease in patients with non-ST-segment elevation acute coronary syndromes," Journal of the American College of Cardiology, vol. 51, no. 10, pp. 991-996, 2008.

[31] A. G. G. Turpie, A. W. A. Lensing, T. Fuji, and D. A. Boyle, "Pharmacokinetic and clinical data supporting the use of fondaparinux $1.5 \mathrm{mg}$ once daily in the prevention of venous thromboembolism in renally impaired patients," Blood Coagulation and Fibrinolysis, vol. 20, no. 2, pp. 114-121, 2009.

[32] K. I. Sombolos, T. K. Fragia, L. C. Gionanlis et al., "Use of fondaparinux as an anticoagulant during hemodialysis: a preliminary study," International Journal of Clinical Pharmacology and Therapeutics, vol. 46, no. 4, pp. 198-203, 2008.

[33] R. M. Kalicki, F. Aregger, L. Alberio, B. Lämmle, F. J. Frey, and D. E. Uehlinger, "Use of the pentasaccharide fondaparinux as an anticoagulant during haemodialysis," Thrombosis and Haemostasis, vol. 98, no. 6, pp. 1200-1207, 2007.

[34] W. E. Dager and T. H. Kiser, "Systemic anticoagulation considerations in chronic kidney disease," Advances in Chronic Kidney Disease, vol. 17, no. 5, pp. 420-427, 2010.

[35] M. M. Samama and G. T. Gerotziafas, "Evaluation of the pharmacological properties and clinical results of the synthetic pentasaccharide (fondaparinux)," Thrombosis Research, vol. 109, no. 1, pp. 1-11, 2003.

[36] C. Klaeffling, G. Piechottka, G. Daemgen-von Brevern et al., "Development and clinical evaluation of two chromogenic substrate methods for monitoring fondaparinux sodium," Therapeutic Drug Monitoring, vol. 28, no. 3, pp. 375-381, 2006.

[37] R. C. Gosselin, J. H. King, K. A. Janatpur, W. H. Dager, E. C. Larkin, and J. T. Owings, "Effects of pentasaccharide
(Fondaparinux) and direct thrombin inhibitors on coagulation testing," Archives of Pathology and Laboratory Medicine, vol. 128, no. 10, pp. 1142-1145, 2004.

[38] L. A. Linkins, J. A. Julian, J. Rischke, J. Hirsh, and J. I. Weitz, "In vitro comparison of the effect of heparin, enoxaparin and fondaparinux on tests of coagulation," Thrombosis Research, vol. 107, no. 5, pp. 241-244, 2002.

[39] A. Smogorzewska, J. T. Brandt, W. L. Chandler et al., "Effect of fondaparinux on coagulation assays: results of College of American pathologists proficiency testing," Archives of Pathology and Laboratory Medicine, vol. 130, no. 11, pp. 16051611, 2006.

[40] F. Depasse, G. T. Gerotziafaz, J. Busson, P. van Dreden, and M. M. Samama, "Assessment of three chromogenic and one clotting assays for the measurement of synthetic pentasaccharide fondaparinux (Arixtra) anti-Xa activity," Journal of Thrombosis and Haemostasis, vol. 2, no. 2, pp. 346-348, 2004.

[41] D. Cook, M. Meade, G. Guyatt et al., "Dalteparin versus unfractionated heparin in critically ill patients," The New England Journal of Medicine, vol. 364, no. 14, pp. 1305-1314, 2011.

[42] M. A. Perazella, "Drug use and nephrotoxicity in the intensive care unit," Kidney International. In press.

[43] G. Cumbo-Nacheli, L. Samavati, and J. A. Guzman, "Bioavailability of fondaparinux to critically ill patients," Journal of Critical Care, vol. 26, no. 4, pp. 342-346.

[44] T. L. Ortel, "Heparin-induced thrombocytopenia: when a low platelet count is a mandate for anticoagulation," Hematology, pp. 225-232, 2009.

[45] T. E. Warkentin, "New approaches to the diagnosis of heparininduced thrombocytopenia," Chest, vol. 127, no. 2, pp. 35S45S, 2005.

[46] M. Prechel and J. M. Walenga, "The laboratory diagnosis and clinical management of patients with heparin-induced thrombocytopenia: an update," Seminars in Thrombosis and Hemostasis, vol. 34, no. 1, pp. 86-96, 2008.

[47] T. E. Warkentin, A. Greinacher, A. Koster, and A. M. Lincoff, "Treatment and prevention of heparin-induced thrombocytopenia: American College of Chest Physicians evidence-based clinical practice guidelines (8th edition)," Chest, vol. 133, no. 6, pp. 340S-380S, 2008.

[48] L. E. Efird and D. R. Kockler, "Fondaparinux for thromboembolic treatment and prophylaxis of heparin-induced thrombocytopenia," The Annals of Pharmacotherapy, vol. 40, no. 7-8, pp. 1383-1387, 2006.

[49] T. E. Warkentin, R. J. Cook, V. J. Marder et al., "Anti-platelet factor 4/heparin antibodies in orthopedic surgery patients receiving antithrombotic prophylaxis with fondaparinux or enoxaparin,” Blood, vol. 106, no. 12, pp. 3791-3796, 2005.

[50] K. H. M. Kuo and M. J. Kovacs, "Successful treatment of heparin induced thrombocytopenia (HIT) with fondaparinux," Thrombosis and Haemostasis, vol. 93, no. 5, pp. 999-1000, 2005.

[51] J. P. J. Wester, A. Leyte, H. M. Oudemans-van Straaten et al., "Low-dose fondaparinux in suspected heparin-induced thrombocytopenia in the critically ill," The Netherlands Journal of Medicine, vol. 65, no. 3, pp. 101-108, 2007.

[52] A. B. Blackmer, M. D. Oertel, and J. M. Valgus, "Fondaparinux and the management of heparin-induced thrombocytopenia: the journey continues," The Annals of Pharmacotherapy, vol. 43, no. 10, pp. 1636-1646, 2009.

[53] B. Lobo, C. Finch, A. Howard, and S. Minhas, "Fondaparinux for the treatment of patients with acute heparininduced 
thrombocytopenia," Thrombosis and Haemostasis, vol. 99, no. 1, pp. 208-214, 2008.

[54] T. E. Warkentin, B. T. Maurer, and R. H. Aster, "Heparininduced thrombocytopenia associated with fondaparinux," The New England Journal of Medicine, vol. 356, no. 25, pp. 2653-2654, 2007.

[55] E. Rota, M. Bazzan, and G. Fantino, "Fondaparinux-related thrombocytopenia in a previous low-molecular-weight heparin (LMWH)-induced heparin-induced thrombocytopenia (HIT)," Thrombosis and Haemostasis, vol. 99, no. 4, pp. 779781, 2008.

[56] K. A. Alsaleh, S. M. A. Al-Nasser, S. M. Bates, A. Patel, T. E. Warkentin, and D. M. Arnold, "Delayed-onset HIT caused by low-molecular-weight heparin manifesting during fondaparinux prophylaxis," American Journal of Hematology, vol. 83, no. 11, pp. 876-878, 2008.

[57] C. J. Lee and J. E. Ansell, "Direct thrombin inhibitors," British Journal of Clinical Pharmacology, vol. 72, no. 4, pp. 581-592, 2011.

[58] J. Trujillo-Santos, J. A. Nieto, A. Ruíz-Gamietea et al., "Bleeding complications associated with anticoagulant therapy in patients with cancer," Thrombosis Research, vol. 125, pp. S58S61, 2010.

[59] S. Vadhan-Raj, "Management of chemotherapy-induced thrombocytopenia: current status of thrombopoietic agents," Seminars in Hematology, vol. 46, no. 2, pp. S26-S32, 2009.

[60] N. Ruíz-Giménez, C. Suárez, R. González et al., "Predictive variables for major bleeding events in patients presenting with documented acute venous thromboembolism. Findings from the RIETE Registry," Thrombosis and Haemostasis, vol. 100, no. 1, pp. 26-31, 2008.

[61] J. A. Nieto, R. Solano, M. D. Ruiz-Ribó et al., "Fatal bleeding in patients receiving anticoagulant therapy for venous thromboembolism: findings from the RIETE registry," Journal of Thrombosis and Haemostasis, vol. 8, no. 6, pp. 1216-1222, 2010.

[62] M. A. Crowther and T. E. Warkentin, "Bleeding risk and the management of bleeding complications in patients undergoing anticoagulant therapy: focus on new anticoagulant agents," Blood, vol. 111, no. 10, pp. 4871-4879, 2008.

[63] M. Franchini, M. Zaffanello, and D. Veneri, "Recombinant factor VIIa. An update on its clinical use," Thrombosis and Haemostasis, vol. 93, no. 6, pp. 1027-1035, 2005.

[64] N. R. Bijsterveld, A. H. Moons, S. M. Boekholdt et al., "Ability of recombinant factor VIIa to reverse the anticoagulant effect of the pentasaccharide fondaparinux in healthy volunteers," Circulation, vol. 106, no. 20, pp. 2550-2554, 2002.

[65] T. Lisman, N. R. Bijsterveld, J. Adelmeijer et al., "Recombinant factor VIIa reverses the in vitro and ex vivo anticoagulant and profibrinolytic effects of fondaparinux," Journal of Thrombosis and Haemostasis, vol. 1, no. 11, pp. 2368-2373, 2003.

[66] G. T. Gerotziafas, F. Depasse, T. Chakroun, M. M. Samama, and I. Elalamy, "Recombinant factor VIIa partially reverses the inhibitor effect of fondaparinux on thrombin generation after tissue factor activation in platelet rich plasma and whole blood," Thrombosis and Haemostasis, vol. 91, no. 3, pp. 531537, 2004

[67] J. Bordes, Y. Asencio, N. Kenane, J. Fesselet, E. Meaudre, and P. Goutorbe, "Recombinant activated factor VII for acute subdural haematoma in an elderly patient taking fondaparinux," British Journal of Anaesthesia, vol. 101, no. 4, pp. 575-576, 2008.

[68] F. Huvers, R. Slappendel, B. Benraad, G. van Hellemondt, and M. van Kraaij, "Treatment of postoperative bleeding after fondaparinux with rFVIIa and tranexamic acid," The Netherlands Journal of Medicine, vol. 63, no. 5, pp. 184-186, 2005.

[69] M. Levi, J. H. Levy, H. F. Andersen, and D. Truloff, "Safety of recombinant activated factor VII in randomized clinical trials," The New England Journal of Medicine, vol. 363, no. 19, pp. 1791-1800, 2010.

[70] A. V. Joshi, P. Mathew, and T. Gore, "Cost-effectiveness of different treatment regimens consisting of NovoSeven Versus FEIBA VH for the home treatment of minor-to-moderate bleeds in hemophilia patients with inhibitors in the United States: a cost-of-bleed model," Journal of Thrombosis and Haemostasis, vol. 3, supplement 1, 2005, abstract no. P2035.

[71] H. R. Buller, A. T. Cohen, B. Davidson et al., "Idraparinux versus standard therapy for venous thromboembolic disease," The New England Journal of Medicine, vol. 357, no. 11, pp. 1094-1104, 2007.

[72] F. F. van Doormaal, A. T. Cohen, B. L. Davidson et al., "Idraparinux versus standard therapy in the treatment of deep venous thrombosis in cancer patients: a subgroup analysis of the Van Gogh DVT trial," Thrombosis and Haemostasis, vol. 104, no. 1, pp. 86-91, 2010.

[73] A. Dao, B. Tuan, and N. Carlson, "Reversal of a potent investigational anticoagulant: idraparinux with recombinant factor VIIa," American Journal of Medicine, vol. 118, no. 10, pp. 1172-1173, 2005.

[74] H. R. Büller, "Efficacy and safety of once weekly subcutaneous idrabiotaparinux in the treatment of patients with symptomatic deep venous thrombosis," Journal of Thrombosis and Haemostasis, vol. 9, no. 1, pp. 92-99, 2011. 


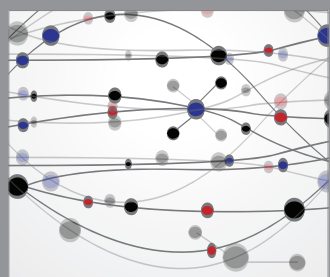

The Scientific World Journal
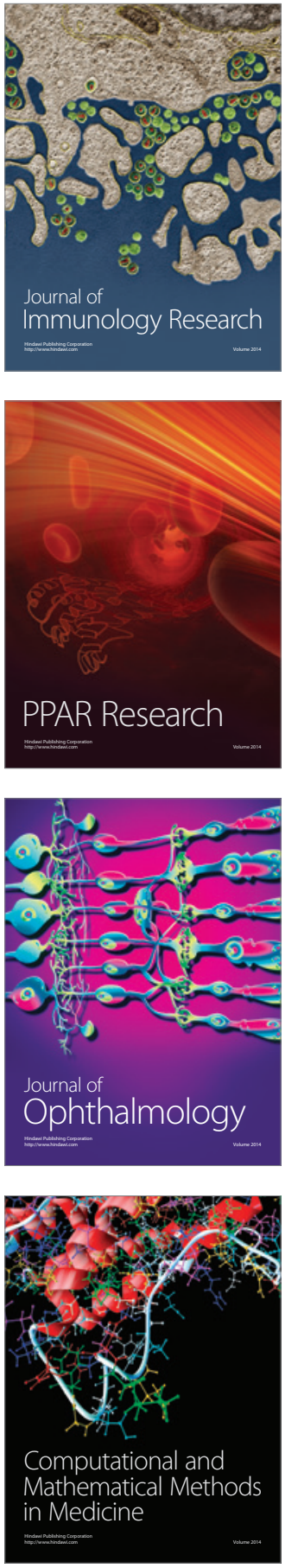

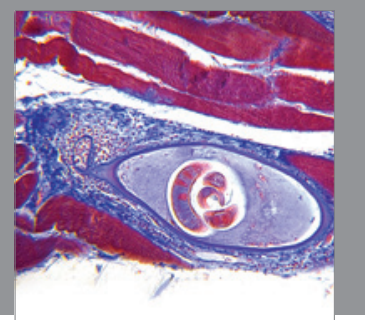

Gastroenterology

Research and Practice
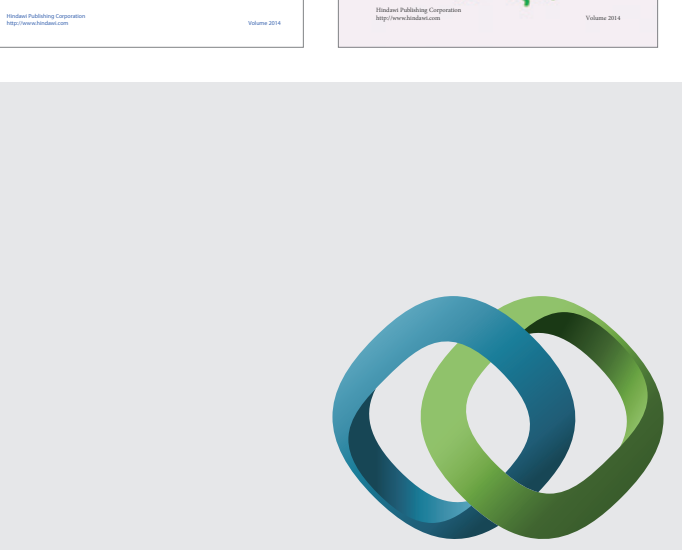

\section{Hindawi}

Submit your manuscripts at

http://www.hindawi.com
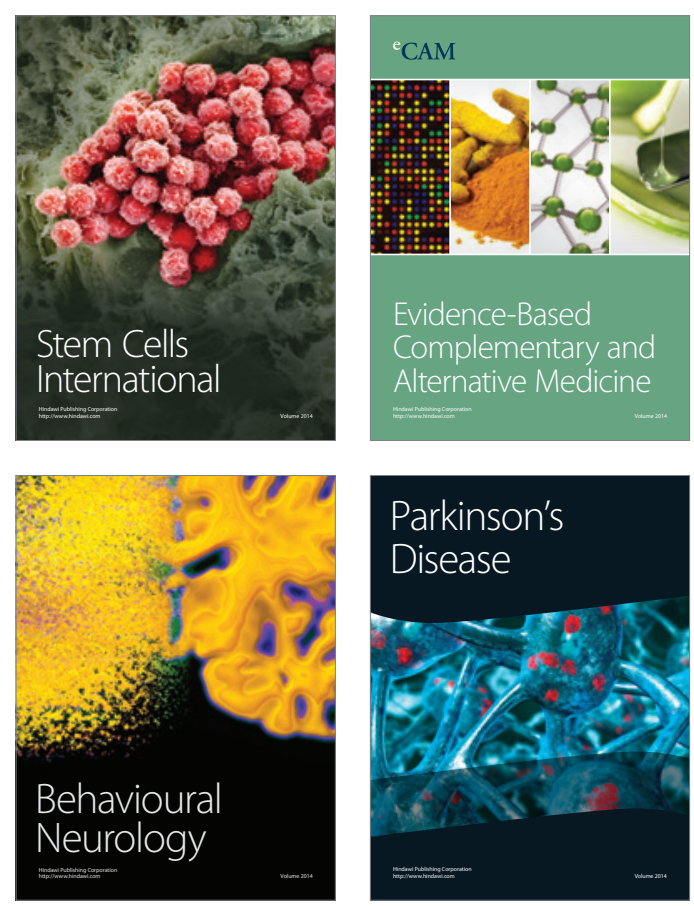

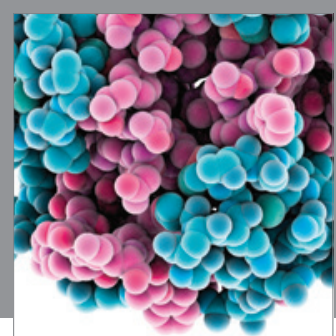

Journal of
Diabetes Research

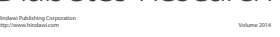

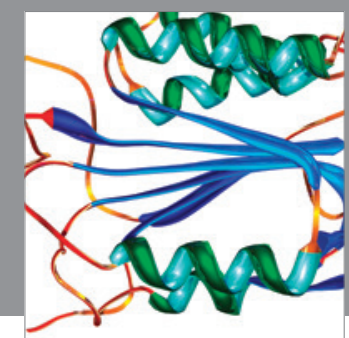

Disease Markers
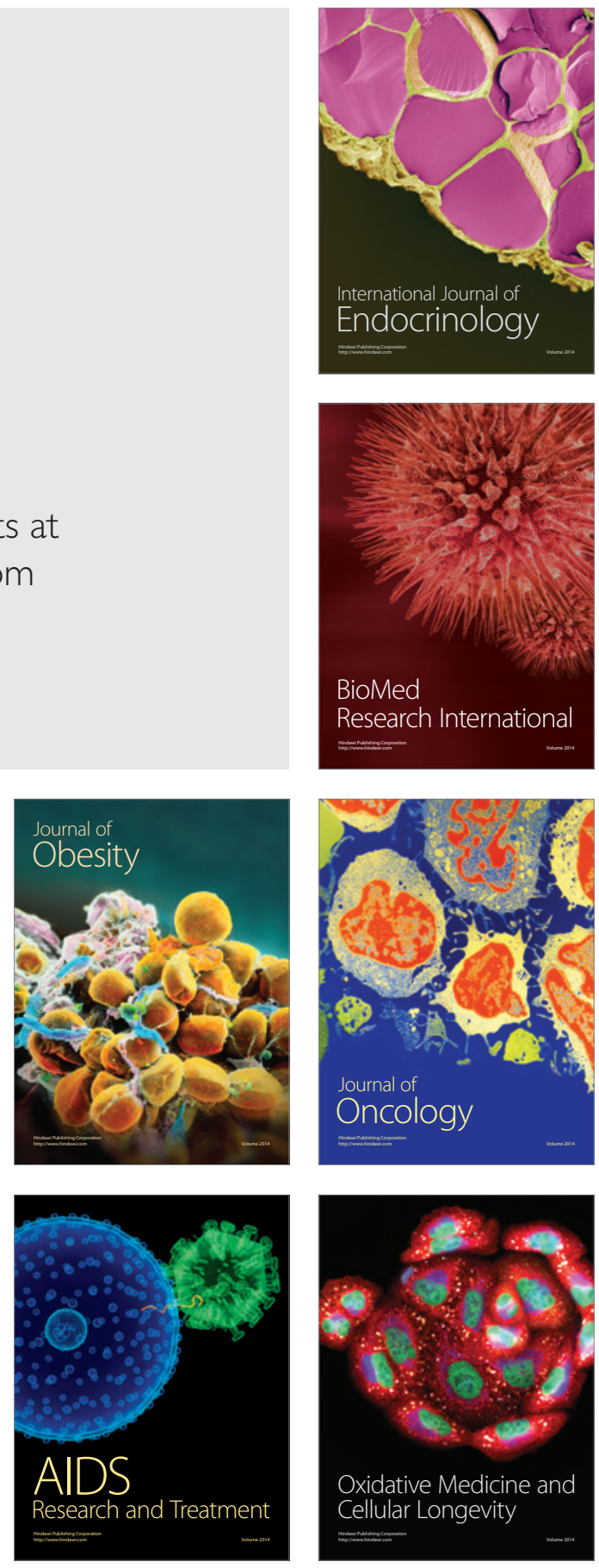